\title{
LARGE-SIGNAL MODELLING AND ANALYSIS OF SWITCHING REGULATORS
}

\author{
ROBERT W. ERICKSON, SLOBODAN ĆUK AND R.D. MIDDLEBROOK
}

\author{
Colifornia Institute of Technology \\ Pasadena, Californio
}

\begin{abstract}
A large-signal switching regulator model is derived, and prominent features of the transient response are determined. In particular, analytical expressions are found for the equilibrium points of the system which yield insight into the large-signal response, and computer-generated transient waveforms are obtained.

As an example, a boost regulator is investigated, and is found to be stable for small signals but unstable for large transients.

\section{INTRODUCTION}

Switching regulators are inherent $1 y$ nonlinear. As a result, it is very difficult to design a stable feedback loop using exact methods since the resulting differential equations cannot generally be solved. Instead, small-signal methods are commonly used, where one linearizes the regulator model about a quiescent operating point. These models are very useful to the practicing engineer since he may apply all of the relatively simple techniques of linear circuit theory such as Bode plots, root locus, etc. The physical insight gained then allows the engineer to intelligently design his feedback loop and to specify important small-signal specifications such as audio susceptibility and output impedance.
\end{abstract}

Unfortunately, because of the small-signal approximation, these methods do not ensure the complete large-signal stability of the quiescent operating point. One might conceive of a regulator which behaves as illustrated in Fig. 1. For small perturbations, less than some radius $r$ from the quiescent operating point, the regulator behaves as predicted by the small-signal model, and transients converge as expected. However, for large perturbations, the nonlinear terms become significant, and some solutions do not converge to the desired quiescent point (i.e., some solutions are unstable). Other large transients do converge, but with a large, distorted waveform which may be much larger than predicted by the small-signal model and hence unacceptable.

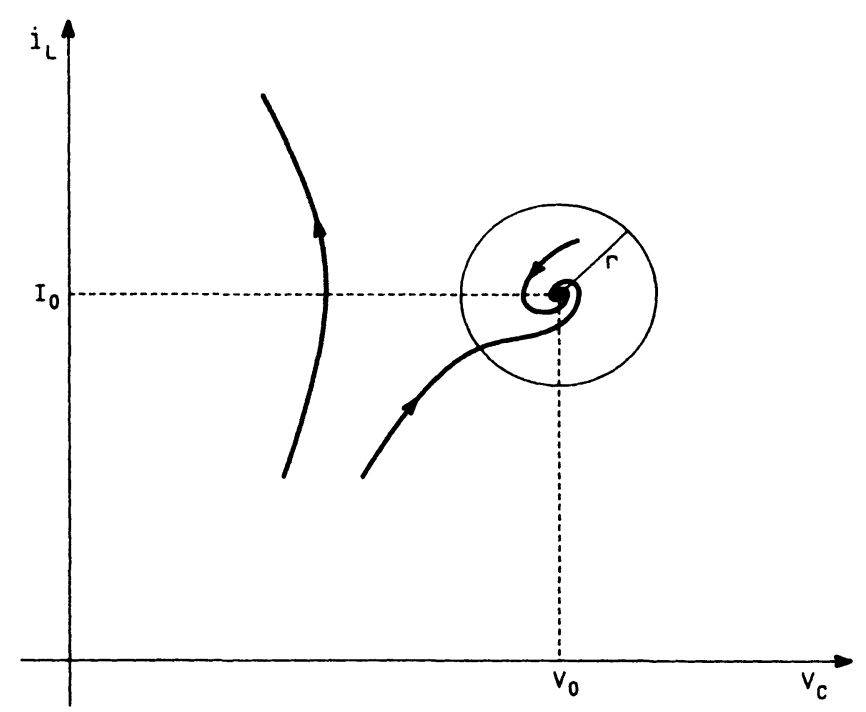

Fig. 1. Hypothetical trajectories of a switching regulator. Inductor current $i_{L}$ is plotted us. capacitor voltage $v_{c}$. A small-signal model was used to design a stable feedback loop; indeed, transient solutions within a radius $r$ of the quiescent operating point $\left(\mathrm{V}_{0}, \mathrm{I}_{0}\right)$ converge as desired. However, transient solutions outside this range may be unstable.

A regulator which exhibits the hypothetical behavior illustrated in Fig. 1 obviously is unreliable and unacceptable. The investigation of the effects of switching regulator nonlinearities and the exposition of some techniques for the avoidance of large-signal instabilities are the subjects of this presentation.

\subsection{The Large-Signal Stability Problem: An Example}

Before embarking on a large-signal analysis, it is necessary before to determine whether the effect of the converter nonlinearity is significant. Is it possible to design a regulator whose response to large perturbations deviates substantially from the response predicted by the small-signal model?

This work was sponsored by the Office of Naval Research, Washington, DC, under contract N00014-78-C-0757. 


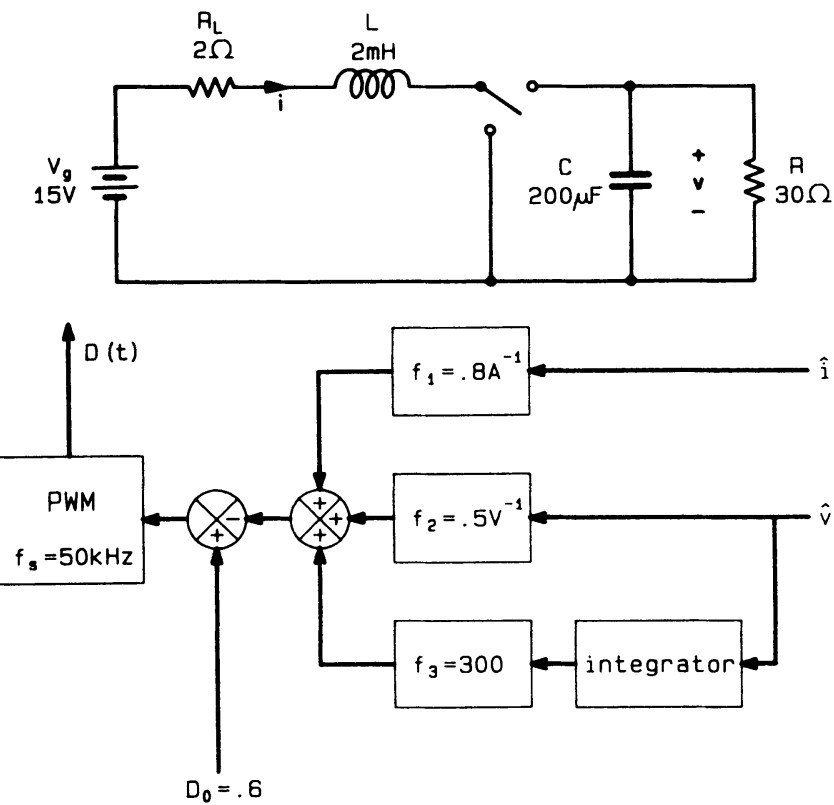

Fig. 2. Three-state boost regulator example.

Even worse, is it possible for the response to be reasonably stable for small signals but diverge for large transients? An example is given in this section which demonstrates that the answer to the above questions is yes; large-signal phenomena exist which can seriously degrade the performance of a switching regulator, and these phenomena are not predicted by small-signal models.

Consider the boost regulator shown in

Fig. 2. The dc gain and efficiency curves are shown in Fig. 3. This regulator was designed to operate at a nominal duty ratio of .6 with an efficiency of $70.5 \%$. The state-space-averaged small-signal model $[1,2,5]$ is shown in Fig. 4 . To stabilize the system in the presence of the right-half-plane zero which appears in the dutyratio-to-output transfer function, the inductor current is fed back in addition to the output voltage. The integral of the output voltage is also fed back to improve regulation. For the values chosen, the closed-loop small-signal response is dominated by two poles with a $Q$ of .6 ; thus, the system appears to be quite stable and nearly critically damped. A computer program, detailed in Fig. 12 later in this paper, was used to investigate the large-signal response of this regulator. The computer-predicted response to a small step change in inductor current and capacitor voltage is diagrammed in Fig. 5. It is indeed well-behaved; the waveforms appear linear, and a very small amount of overshoot occurs in the control ( $\hat{d})$ waveform. The small-signal model is an excellent approximation in this case.

The response to a step change of intermediate proportions is shown in Fig. 6 . The solid line is the nonlinear response, and the dotted line is the response predicted by the small-signal model. Although this response is (a)

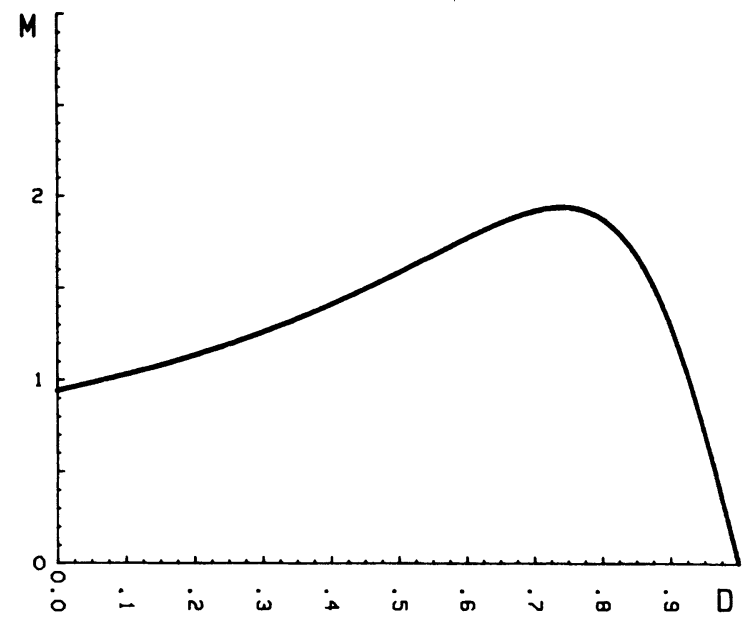

(b)

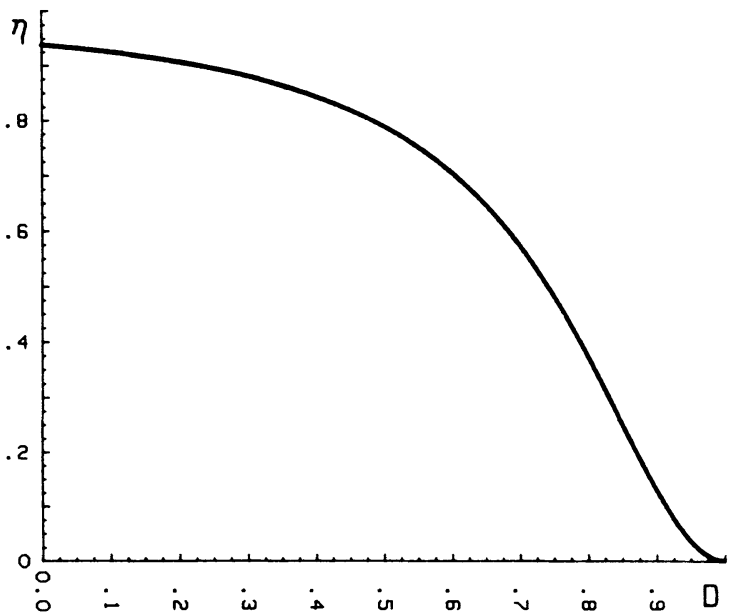

Fig. 3. $D_{c}$ analysis of the boost example: (a) Equilibrium gain $\mathrm{M}=\mathrm{v}_{\mathrm{c}} / \mathrm{v}_{\mathrm{g}} ;(\mathrm{b})$ Equilibrium efficiency $n$.
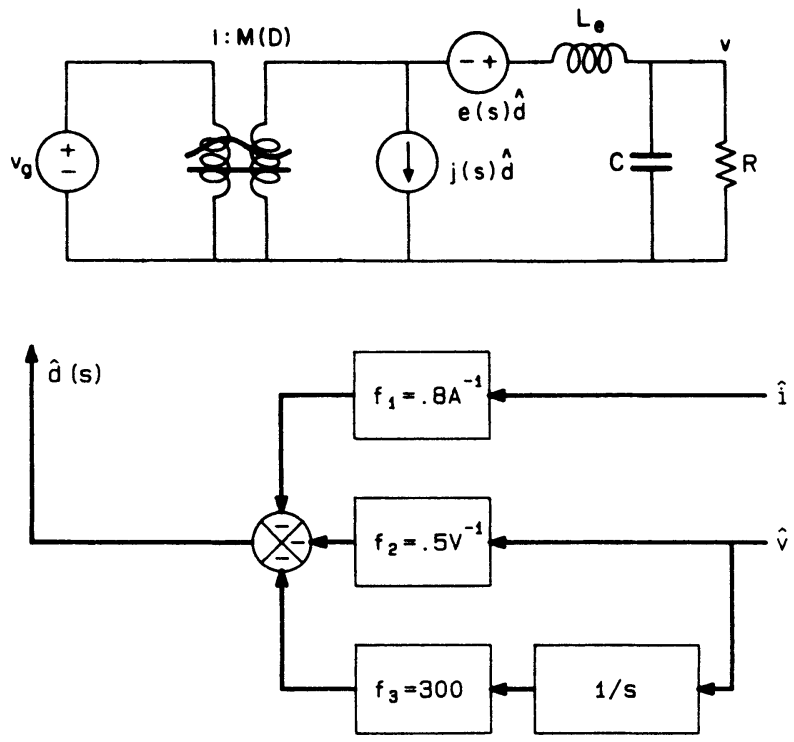

Fig. 4. Small-signal state-space averaged model used to design the feedback loop of the three-state boost regulator example. 


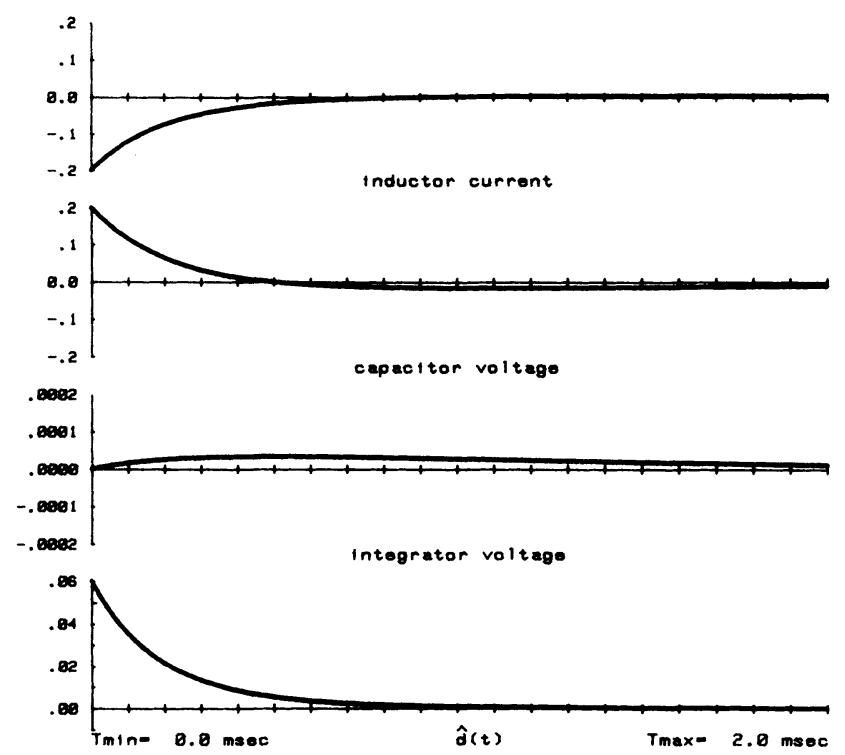

Fig. 5. Computer-generated time domain waveforms of the response to a small perturbation in capacitor voltage $\hat{v}$ and inductor current $\hat{i}$. The small-signal approximation is justified in this case, and the response converges as predicted.

stable, it is decidedly nonlinear. The peak inductor current is larger than the amount predicted by the small-signal model, and the control (d) waveform overshoots its nominal value much more than expected. The small-signal approximation is unjustified in this case.

The response to a yet larger step change in inductor current and capacitor voltage is shown in Fig. 7. The system is unstable in this case! The voltages and currents in the system become large, and the control signal saturates at its maximum value. Obviously, this design is unreliable.

In this example, the small-signal approximation is unjustified for moderate or large perturbations. Hence, even though the small-signal model predicts that the system response is welldamped, the effect of the nonlinearity can be of significance, causing larger overshoots than expected and possibly even instabilities.

\subsection{Outline of Discussion}

From the above example, it is apparent that small-signal switching regulator models do not necessarily include all features of importance. It is of interest, therefore, to model these additional large-signal effects. In Section 2, a nonlinear model is derived which predicts the behavior described in the boost example above. Two versions of this model are described. First, a discrete-time model is derived which is well-suited for computer simulations. Second, a continuous-time model is found which is sometimes more convenient for analytical calculations.

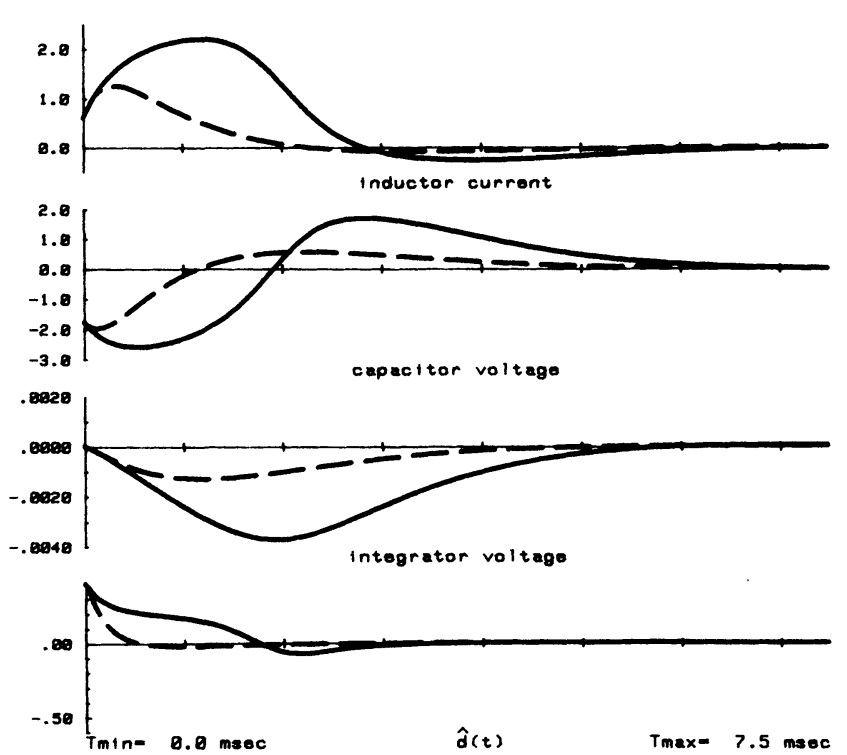

Fig. 6. Computer-generated waveforms of the response to a perturbation of intermediate proportions. Dashed line: as predicted by the small-signal model. Solid line: as predicted by the complete large-signal nonlinear model described in Fig. 12. Peak voltage and current values are significantly larger than the amounts predicted by the small-signal model.

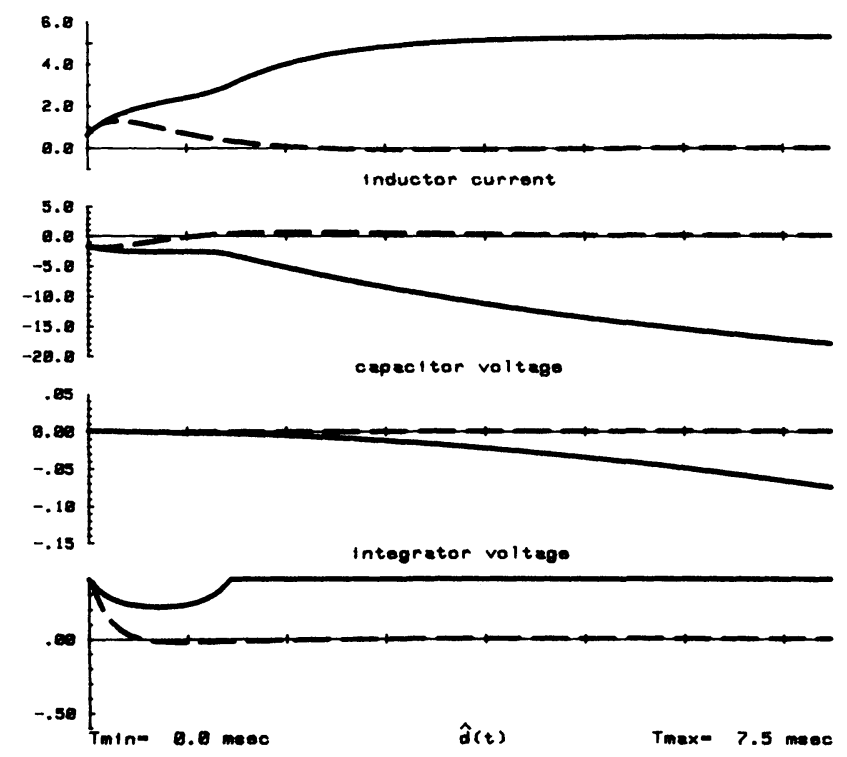

Fig. 7. Computer-generated waveforms of the response to a large perturbation. Dashed line: as predicted by the small-signal model. Solid line: as predicted by the large-signal nonlinear model. The system is unstable.

Next, the saturation of the pulse-width modulator is accounted for. This phenomenon has a significant effect on the large-signal response of the regulator, and hence cannot be ignored. Models are derived which describe the response of the regulator in the saturated regions. 
In Section 3, the implications of these models are examined. First, the equilibrium points of the system are calculated. The presence of real equilibrium points in addition to the desired quiescent operating point indicates the existence of unstable transient solutions; therefore, these additional real equilibrium points must be eliminated. Next, the trajectories or transient waveforms are calculated. Peak transient current and voltage levels may then be determined, and the existence or absence of unstable solutions verified.

The entire procedure is summarized in Section 4 .

\section{LARGE-SIGNAL MODELLING}

In this section, the nonlinear state equations which describe switching regulators operating in the continuous conduction mode are derived, and the regions of their validity are identified. Two versions of the model are useful. First, a discrete-time model is found which is suitable for computer simulation. Second, an additional approximation is made which yields a continuous-time model; this model is often more convenient for analytical calculations. Both models incorporate the key "linear-ripple approximation" used in previous small-signal methods $[1,2,3,4,5]$. However, no small-signal assumption is made; as a result, the method is valid for large signals.

Next, the saturation characteristics of the pulse-width modulator are accounted for. The duty ratio is always restricted to lie in a range no greater than $[0,1]$, and the effect of this restriction on the stability of the regulator is so profoimd that no large-signal analysis can ignore it. The result of this section is the formulation of basic state equations which model the switching regulator and which are used in the subsequent sections to determine stability regions and peak transient component stress levels.

\subsection{Unsaturated Region Mode1}

The first step is to write the state equations of the regulator system during the two switched intervals $\mathrm{DT}_{\mathbf{S}}$ and $\mathrm{D}^{\prime} \mathrm{T}_{\mathbf{S}}$. During each interval, the system may be described by a set of linear differential equations:

during interval $\mathrm{DT}_{S}$ (transistor $\mathrm{ON}$ )

$$
K \frac{d \underline{x}(t)}{d t}=A_{1} \underline{x}(t)+B_{1} \underline{u}
$$

during interval $\mathrm{D}^{\prime} \mathrm{T}_{S}$ (transistor $\mathrm{OFF}$ )

$$
K \frac{d \underline{x}(t)}{d t}=A_{2} \underline{x}(t)+B_{2} \underline{u}
$$

where $D=$ duty ratio, $D^{\prime}=1-D$.

$T_{S}=$ time of one complete switching period.

$\mathrm{K}$ is a matrix usually containing values of inductance and capacitance.

$\underline{x}$ is a state vector, usually comprised of inductor currents and capacitor voltages.

$\underline{u}$ is a vector of independent sources.

These equations may be solved exactly; however, the subsequent analysis is greatly simplified if the "linear-ripple approximation" $[1,2,3,4,5]$ is made. Specifically, if the natural frequencies $\omega_{i}$ of the converter power stage are all well below the switching frequency, then Eqs. (1) and (2) above have approximately linear solutions. This is indeed the case in welldesigned converters, in which the switching ripple is small. In this case, only the terms to order $\left(\omega_{i} T_{s}\right)$ need be considered; higher-order terms are negligible.

The result of this approximation is the following solutions for $\underline{x}(t)$ during the two switched intervals:

$$
\begin{aligned}
& \underline{x}(t)=\left(I+A_{1} t\right) \underline{x}(0)+B_{1} \underline{u t} \\
& \text { during } 0 \leq t \leq D_{s} \\
& \underline{x}(t)=\left(I+A_{2}\left(t-D T_{s}\right) \underline{x}\left(D_{s}\right)+B_{2} \underline{u}_{s}\left(t-D T_{s}\right)\right. \\
& \text { during } D_{s} \leq t \leq T_{s}
\end{aligned}
$$

Combination of Eqs. (3) and (4), and elimination of the second-order terms which appear, yields the following expression for $\underline{x}\left(T_{s}\right)$ :

$$
\underline{x}\left(T_{s}\right)=\left[I+T_{s}\left(D A_{1}+D^{\prime} A_{2}\right)\right] \underline{x}(0)+T_{s}\left(D_{1}+D^{\prime} B_{2}\right) \underline{u}
$$

Furthermore, after $n+1$ switching intervals,

$$
\begin{aligned}
\underline{x}\left((n+1) T_{s}\right)=\underline{x}_{n+1} & =\left[I+T_{s}\left(D_{n} A_{1}+D_{n}^{\prime} A_{2}\right)\right]_{n} \\
& +T_{s}\left[D_{n} B_{1}+D_{n}^{\prime} B_{2}\right] \underline{u}
\end{aligned}
$$

This is the basic difference equation which describes the response of the system. It contains a constant term; hence, the system is not in equilibrium at the origin. It is convenient in the analysis which follows to redefine the axes so that the quiescent operating point of the regulator is at the origin. The quiescent operating point occurs at:

$$
\underline{x}_{n+1}=x_{n}=\underline{x}_{0}, \quad D_{n+1}=D_{n}=D_{0}
$$


Insertion of Eq. (7) into Eq. (6) and solution for $\underline{x}_{0}$ yields

$$
\underline{x}_{0}=-\left(D_{0} A_{1}+D_{0}^{\prime} A_{2}\right)^{-1}\left(D_{0} B_{1}+D_{0}^{\prime} B_{2}\right) \underline{u}
$$

The axes may now be shifted by use of the substitution below.

$$
\begin{aligned}
\underline{x}_{n} & =\underline{x}_{0}+\underline{\hat{x}}_{n}, D_{n}=D_{0}+\hat{d}_{n} \\
\underline{x}_{n+1} & =\left[I+T_{s}\left(D_{0} A_{1}+D_{0}^{\prime} A_{2}\right)\right] \underline{\hat{x}}_{n}+T_{s}\left[\left(A_{1}-A_{2}\right) \underline{x}_{0}+\left(B_{1}-B_{2}\right) \underline{u}_{n}\right] \hat{d}_{n} \\
& +T_{s} \hat{d}_{n}\left(A_{1}-A_{2}\right) \underline{\hat{x}}_{n}
\end{aligned}
$$

Eq. (10) arises from the substitution of Eq. (9) into Eq. (6), and is the difference equation which describes the regulator while operating in the normal, unsaturated mode. No small-signal assumption has been made; hence, Eq. (10) is valid for large signals.

The duty ratio is usually a linear function of the regulator states. In this case,

$$
\hat{\mathrm{d}}_{\mathrm{n}}=-\underline{\mathrm{f}}^{\mathrm{T}} \hat{\mathrm{x}}_{\mathrm{n}}
$$

where $\underline{\mathrm{f}}=$ feedback gain vector.

As a result of the linear dependence of $\hat{d}_{n}$ on $\hat{x}_{n}$, the $T_{s} \hat{d}_{n}\left(A_{1}-A_{2}\right) \hat{x}_{n}$ term in Eq. (10) is quadratic, and the difference equation is nonlinear. This nonlinearity can seriously degrade the transient response of the regulator, possibly causing instabilities.

The discrete form of Eqs. (10) and (11) makes them ideally suited for evaluation by computer. It is a simple matter to implement these equations on a small desktop computer and obtain the largesignal transient response of any switching regulator operating in the unsaturated region. In view of the difficulty of obtaining a closedform analytical solution to Eq. (10) under transient conditions, this is an attractive alternative. The time domain waveforms iliustrated in this paper were obtained in this manner.

Although the discrete equations above are well-suited for digital computer evaluation, they are sometimes inconvenient for analytical

calculations. It is then preferred to obtain a continuous-time model which contains familiar $R^{\prime} s$, L's, C's, and nonlinear devices, and hence yields physical insight into the design problem. This has previously been accomplished for the smallsignal case [1], and the same technique is applicable here. In particular, one uses the Euler forward-differencing approximation to estimate the continuous-time derivative of the state vector, as below:

$$
\frac{\mathrm{d} \underline{\underline{x}}(t)}{d t} \simeq \frac{\frac{\hat{x}_{n+1}-\hat{x}_{n}}{T_{s}}}{T_{n+1}}
$$

This approximation is valid if all natural frequencies of the closed-loop regulator are much less than the switching frequency. With this approximation, Eq. (10) becomes

$$
\begin{gathered}
\frac{d \underline{\hat{x}}(t)}{d t}=\left(D_{0} A_{1}+D_{0}^{\prime} A_{2}\right) \underline{\hat{x}}(t)+\left[\left(A_{1}-A_{2}\right) \underline{x}_{0}+\left(B_{1}-B_{2}\right) \underline{u}\right] \hat{d}(t) \\
+\hat{d}(t)\left(A_{1}-A_{2}\right) \underline{\hat{x}}(t)
\end{gathered}
$$

with $\hat{d}(t)=-\underline{f}^{T} \underline{\hat{x}}(t)$

This is a continuous-time state equation which describes the regulator while operating in the normal, unsaturated mode. It can be used to infer the nature of the response to large perturbations and the existence of instabilities. Owing to the presence of the $\hat{d}\left(A_{1}-A_{2}\right) \hat{x}$ term, this state equation contains quadratic nonlinear terms.

Thus, the equations which describe the response of switching regulators during large transients have been found for the case where the pulse-width modulator is unsaturated and the system operates in its usual mode. The linearripple approximation was made; this has the desirable effect of simplifying the analysis while ignoring the usually negligible effect of the high frequency switching ripple. A set of discrete state equations with quadratic nonlinearities is the result; these equations are well-suited for computer evaluation of the large-signal transient response. An additional approximation may be made which yields a continuous-time version of the state equations. This is of ten desirable for analytical design.

\subsection{Saturated Region Mode1s}

So far, it has been assumed that the regulator always operates in its usual unsaturated mode. No account has yet been made of the inherent limits on the duty ratio: the duty ratio must always lie within the range $[0,1]$; often, the limit is even more restrictive. Outside the usual operating range, the duty ratio remains constant at its saturated value, and the regulator behaves as an open-loop linear system. This can significantly affect the large-signal stability of the regulator: in consequence, these additional modes of operation cannot be ignored. Some aspects of this effect have been previously considered for the buck regulator $[6,7]$; the phenomenon is further investigated here.

As an example, consider the two-state boost regulator of Fig. 8. In order to stabilize the system in the presence of the right-half plane zero which appears in the duty-ratio-to-output transfer function, the inductor current is fed back in addition to the output voltage. The 


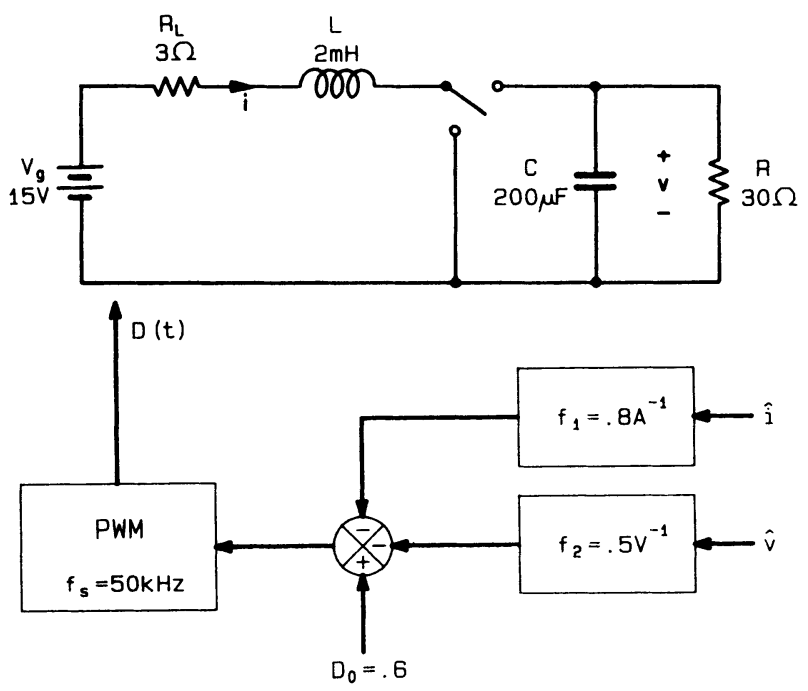

Fig. 8. Two-state boost regulator example.

expression for the control signal (duty ratio) is therefore

$$
\begin{aligned}
& D(t)=D_{0}+\hat{d}(t) \\
& \hat{d}(t)=-f_{1} \hat{i}_{L}-f_{2} \hat{v}_{c}=-\underline{f} \underline{\hat{x}}
\end{aligned}
$$

where $\mathrm{D}_{0}=$ quiescent duty ratio

$$
\begin{aligned}
& \mathrm{D}_{0}=\text { quiescent duty ratio } \\
& \mathrm{f}_{1}=\text { current feedback gain } \\
& \mathrm{f}_{2}=\text { voltage feedback gain }
\end{aligned}
$$

If $D(t)$ is limited to the range $\left[D_{\min }, D_{\max }\right]$ then the unsaturated region is the section of the phase where

$$
\mathrm{D}_{\min }<\mathrm{D}_{0}-\underline{\mathrm{f}}^{\mathrm{T}} \underline{\hat{\mathrm{x}}}<\mathrm{D}_{\max }
$$

To the left of this region, the duty ratio is fixed and equal to $D_{\max }$, and to the right of this region, the duty ratio is fixed and equal to $D_{\min }$. The situation is illustrated in Fig. 9.

The state equations in the saturated regions are easily found. When $-\underline{\mathrm{f}} \hat{\mathrm{x}}$ is greater than $D_{\max }-D_{0}$, then $D(t)$ is constant and equal to $D_{\max }$. Likewise, $D(t)$ is constant and equal to $D_{\min }$ when $-\underline{f}^{\mathrm{T}} \hat{\underline{x}}$ is less than $D_{\text {min }}-D_{0}$. Substitution of $D_{n}=D_{\text {sat }}$ into Eq. (6) yields

$$
\begin{aligned}
\underline{x}_{n+1}=[I & \left.+T_{s}\left(D_{\text {sat }} A_{1}+D_{\text {sat }}^{\prime} A_{2}\right)\right]_{x_{n}}+T_{s}\left[D_{\text {sat }} B_{1}+D_{\text {sat }}^{\prime} B_{2}\right] \underline{u} \\
\text { where } D_{\text {sat }} & =\text { either } D_{\max } \text { or } D_{\text {min }} \\
D_{\text {sat }}^{\prime} & =1-D_{\text {sat }}
\end{aligned}
$$

This is a system of linear difference equations which describe the response of the system in the saturated regions.

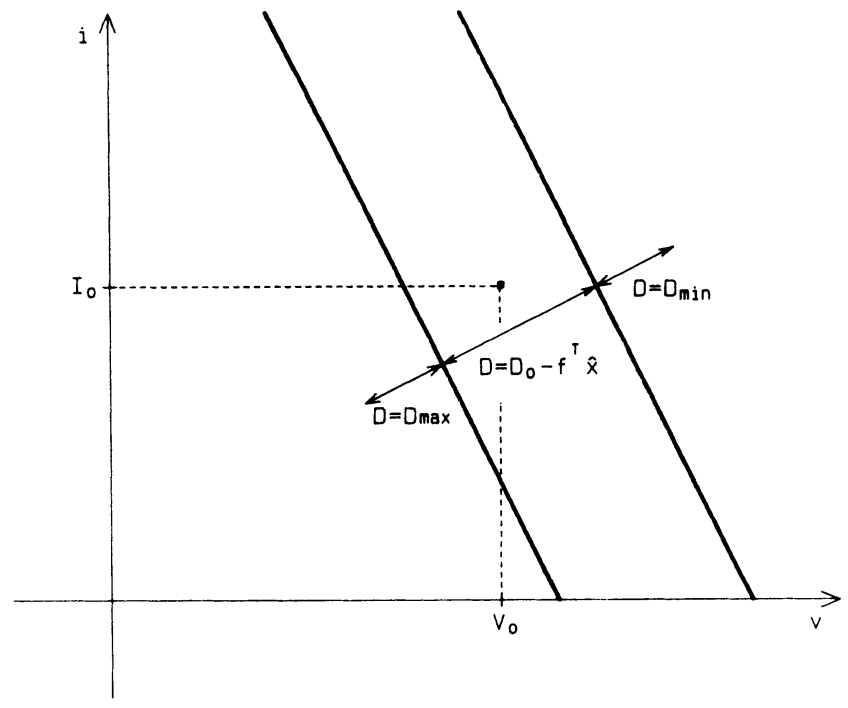

Fig. 9. The saturated and unsaturated regions of the state plane. $\mathrm{I}_{0}$ and $\mathrm{V}_{0}$ are the quiescent inductor current and capacitor voltage.

The continuous-time state equations are again found by use of the forward-differencing approximation. The result for the $D=D_{\text {sat }}$ saturated region is

$$
\begin{aligned}
\frac{d \underline{\mathrm{x}}(t)}{d t} & =\left[D_{\text {sat }} A_{1}+D_{\text {sat }}^{\prime} A_{2}\right] \underline{x}(t) \\
& +\left[D_{\text {sat }} B_{1}+D_{\text {sat }}^{\prime} B_{2}\right] \underline{u}
\end{aligned}
$$

This is a system of linear differential equations which describes the response in the $\mathrm{D}=\mathrm{D}_{\text {sat }}$ saturated region. Since the systems described by Eq. (16) and (17) are linear their solutions are exponential in nature. Furthermore, they contain exactly one equilibrium point. This point occurs at

$$
\underline{x}_{0}=-\left[D_{\text {sat }} A_{1}+D_{\text {sat }}^{\prime} A_{2}\right]^{-1} \cdot\left[D_{\text {sat }} B_{1}+D_{\text {sat }}^{\prime} B_{2}\right] \underline{u}
$$

This equilibrium point may or may not occur within the saturated region.

Thus, three modes of operation have been identified. This change of operating mode occurs owing to the saturation of the pulse-width modulation process. The regions in the state plane in which each mode occurs have been found, and the relevant state equations have been cierived. In the next section, this knowledge is applied to the study of the large-signal transient waveforms and instabilities which are likely to occur in switching regulators. 


\section{LARGE-SIGNAL ANALYSIS}

The objective of this section is the construction of the system response and the identification of sources of potential instability. First, the equilibrium points of the system arcalculated. It is possible for more than one equilibrium point to exist, and this can lead to instability under large transient conditions. second, the trajectories of the states of the regulator are determined, either by hand or by computer. The salient features are identified, and it then becomes apparent how to modify the system in order to obtain an acceptable response. The analysis is demonstrated on the boost regulator of Fig. 8 .

\subsection{Equilibrium Points}

The equilibrium points of a system are the most prominent features of the state-plane portrait of a nonlinear system. Calculation of the equilibrium points is a useful tool for pinpointing the source of instabilities and for constructing trajectories.

Two types of equilibrium points can exist for a given region of the state plane. The first type, known as a "real equilibrium point," is an equilibrium point which occurs inside the given region. The presence of real equilibrium points in addition to the desired quiescent operating point guarantees the presence of at least one unstable solution. Hence, such points must be avoided. The second type is called a "virtual equilibrium point" and occurs outside the given region. The system is not actually in equilibrium at a virtual equilibrium point; nonetheless, these points can influence the transient response of the regulator. The presence of virtual equilibrium points guarantees neither the existence nor the absence of unstable transient responses.

Equilibrium points occur where

$$
\begin{array}{ll}
\hat{\mathrm{x}}_{\mathrm{n}+1}^{*}=\hat{\mathrm{x}}_{\mathrm{n}}^{*} & \text { (discrete) } \\
\frac{\mathrm{d} \hat{\mathrm{x}}^{*}}{\mathrm{dt}}=0 & \text { (continuous) }
\end{array}
$$

Since the Euler forward differencing approximation, Eq. (12), has been used, Eqs. (19a) and (19b) are equivalent.

Insertion of Eq. (19b) into Eq. (13) yields

$$
\underline{0}=\left[\mathrm{A}+\mathrm{d}^{*} \mathrm{~B}\right] \underline{\hat{\mathrm{x}}}^{*}
$$

where $A=D_{0} A_{1}+D_{0}^{\prime} A_{2}-\left(A_{1}-A_{2}\right) \underline{x}_{0} f^{T}-\left(B_{1}-B_{2}\right) \underline{u} \underline{f}^{T}$

$$
\begin{aligned}
& B=A_{1}-A_{2} \\
& \mathrm{~d}^{*}=-\underline{\mathbf{f}}^{\mathrm{T}} \underline{\hat{\mathrm{x}}}^{*} \\
& \underline{\hat{x}}^{*}=\text { the value of } \underline{\hat{x}} \text { at the desired }
\end{aligned}
$$

$A$ is the small-signal continuous-time closed-loop system matrix, and $B$ is a matrix which describes the nonlinear term. Equation (20) describes the positions of the equilibrium points in the unsaturated region. In addition to the trivial solution $\hat{\mathrm{x}}^{*}=\underline{0}$ (the desired quiescent operating point), a number of extra solutions may exist. One may easily find these other equilibrium points by first solving Eq. (21)for $d^{*}$, the value of the control ( $\hat{\mathrm{d}})$ at $\mathrm{x}^{*}$.

$$
\operatorname{det}\left[A+d^{*} B\right]=0
$$

Once the values of $\mathrm{d}^{*}$ are known, the solution of Eq. (20) for $x^{*}$ is straightforward. One can then see how to design the regulator such that these additional equilibrium points lie sufficiently far outside of the unsaturated region. Their influence on the transient response of the regulato $i$ can then be made small.

For the boost example of Fig. 8, the quantities in Eq. (20) are

$$
\begin{aligned}
& \underline{\hat{x}}^{*}=\left[\begin{array}{l}
\hat{\mathrm{i}}^{*} \\
\hat{v}^{*}
\end{array}\right] \\
& \underline{f}=\left[\begin{array}{l}
\mathrm{f}_{1} \\
\mathrm{f}_{2}
\end{array}\right]
\end{aligned}
$$$$
\begin{aligned}
& A=\left[\begin{array}{cc}
-R_{1} & -n_{1} \\
n_{2} & -1 / R_{2}
\end{array}\right] \\
& B=\left[\begin{array}{cc}
0 & 1 \\
-1 & 0
\end{array}\right]
\end{aligned}
$$

$$
\begin{aligned}
& \text { where } \mathrm{R}_{1}=\mathrm{R}_{\mathrm{L}}+\mathrm{v}_{0} \mathrm{f}_{1} \quad \mathrm{n}_{1}=\mathrm{D}_{0}^{\prime}+\mathrm{v}_{0} \mathrm{f}_{2} \\
& \mathrm{n}_{2}=\mathrm{D}_{0}^{\prime}+\mathrm{I}_{0} \mathrm{f}_{1} \quad \mathrm{R}_{2}=\mathrm{R} \|\left(-\mathrm{I}_{0} \mathrm{f}_{2}\right) \\
& \mathrm{I}_{0}=\text { quiescent inductor current } \\
& \mathrm{v}_{0}=\text { quiescent output voltage }
\end{aligned}
$$

Eq. (21), the expression for the control ( $\left.d^{*}\right)$ at the equilibrium points, then becomes

$$
\mathrm{d}^{*^{2}}-\mathrm{d}^{*}\left(\mathrm{n}_{1}+\mathrm{n}_{2}\right)+\mathrm{n}_{1} \mathrm{n}_{2}+\frac{\mathrm{R}_{1}}{\mathrm{R}_{2}}=0
$$

This equation is quadratic and has two roots; hence, two equilibrium points may exist for the unsaturated region in addition to the quiescent operating point $\underline{\hat{x}}=\underline{0}$. For the values specified in Fig. 8, the two roots are

$$
d^{*}=.236,13.64
$$

The equilibrium point at $\mathrm{d}^{*}=.236$ is we11 within the unsaturated region. It causes the large-signal transient response to differ significantly from that predicted by small-signal models; in fact, some solutions are unstable. 
The root $d^{*}=13.64$ represents a virtual equilibrium point. It lies outside the unsaturated region where Eq. (23) is valid; in consequence, the system is not actually in equilibrium at this point. Nonetheless, it is possible for a virtual equilibrium point to influence the response of a nonlinear system. In this case, however, the distance it lies away from the unsaturated region is sufficiently large that its effect on the trajectories is negligible.

The positions in the state plane of the equilibrium points are now found by solution of Eq. (20). For this example, one obtains

$$
\begin{aligned}
& \hat{i}^{*}=-\frac{d^{*}}{f_{1}+R_{2} \hat{f}_{2}\left(n_{2}-d^{*}\right)} \\
& \hat{v}^{*}=-\frac{d^{*}}{f_{2}+\frac{f_{1}}{R_{1}}\left(d^{*}-n_{1}\right)}
\end{aligned}
$$

For the values specified in Fig. 8, these expressions yield

$$
\begin{aligned}
& \hat{i}^{\star}=2.0 \mathrm{~A}, \hat{\mathrm{v}}^{*}=-3.7 \mathrm{~V} \text { at } \mathrm{d}^{\star}=.236 \\
& \hat{\mathrm{i}}^{*}=1.9 \mathrm{~A}, \hat{\mathrm{v}}^{*}=-24.2 \mathrm{~V} \text { at } \mathrm{d}^{*}=13.64
\end{aligned}
$$

The unsaturated region equilibrium points are summarized in Fig. 10. Two real equilibrium points exist: in addition to the desired quiescent operating point at $\hat{x}=0$, an unwanted real equilibrium point occurs as shown; hence, unstable responses are expected to occur. A virtual equilibrium point also occurs, but has little effect on the response of the system.

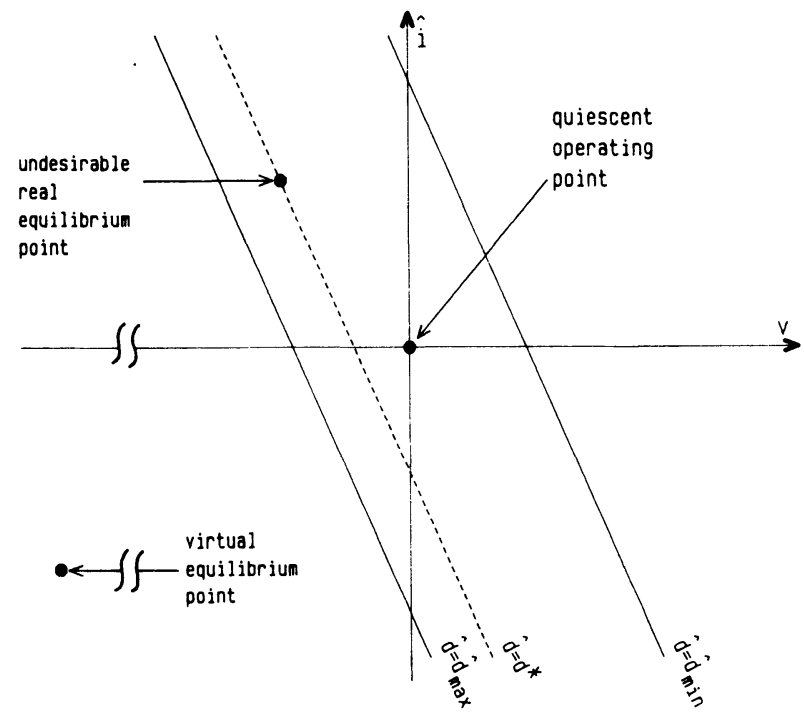

Fig. 10. Summary of the unsaturated region equilibrium points for the boost example in Fig. 8. In addition to the usual quiescent operating point, a real equilibrium point exists at $\mathrm{d}^{*}=.236$. A (insignificant) virtual equilibrium point occurs at $\mathrm{d}^{*}=13.64$.
The next step is the investigation of the equilibrium points of the saturated regions. Since in these regions the response is linear, exactly one equilibrium point occurs for each region. If either of these points is real, then unstable responses exist. Therefore, the equilibrium points of both saturated regions must be virtual in a globally-stable regulator.

The equilibrium point for the $D=D_{\max }$ saturated region is given by Eq. (18); a similar expression can be found for the $D=D_{\min }$ saturated region. This equation is now solved to find the positions of the saturated region equilibrium points. For the boost example, one obtains

$$
\begin{aligned}
& v_{\text {sat }}=\frac{v_{g}}{D_{\text {sat }}^{\prime}} \cdot \frac{1}{1+R_{L} / D_{\text {sat }}^{\prime 2} R} \\
& I_{\text {sat }}=\frac{V_{\text {sat }}}{D_{\text {sat }}^{\prime} R}
\end{aligned}
$$

where

$$
\begin{aligned}
\mathrm{V}_{\text {sat }}= & \text { equilibrium output voltage for } \mathrm{D}=\mathrm{D}_{\text {sat }} \\
& \text { saturated region } \\
\mathrm{I}_{\text {sat }}= & \text { equilibrium induct or current for } \\
& \mathrm{D}=\mathrm{D}_{\text {sat }} \text { saturated region. }
\end{aligned}
$$

One possible design strategy is to limit the duty ratio to a range sufficiently smaller than $[0,1]$, thereby improving the response by moving the saturated region equilibrium points well outside of their respective regions. Hence, it is of interest to determine the locus of $V_{\text {sat }}$ and $I_{\text {sat }}$ in the state plane for various values of $D_{\text {sat }}$. Combination of Eqs. (27) and (28) yields

$$
I_{\text {sat }}^{2} R R_{L}-I_{\text {sat }} R_{g}+V_{\text {sat }}^{2}=0
$$

Thus, the locus is an ellipse. Eq. (29) is plotted in Fig. 11. It can be seen that the $D=0$ point is well outside of the $D=D_{\text {min }}$ saturated region; consequently, the choice of $\mathrm{D}_{\min }=0$ poses no apparent problems. However, the $\mathrm{D}=1$ point lies inside the $D=D_{\max }$ saturated region for the case illustrated. As a result, unstable solutions are guaranteed to exist for the choice of $D_{\max }=1$.

One way to avoid obtaining a real equilibrium point in the $D=D_{\max }$ saturated region is to choose $D_{\max }$ sufficiently less than one, thereby moving the equilibrium point outside the region. A second way is to lower the ratio $f_{2} / f_{1}$ (i.e., increase the amount of current feedback in relation to the output voltage feedback). This moves the $D=D_{\max }$ saturated region boundary to the left, past the equilibrium point. Both strategies are effective in eliminating the presence of unstable transient solutions. 


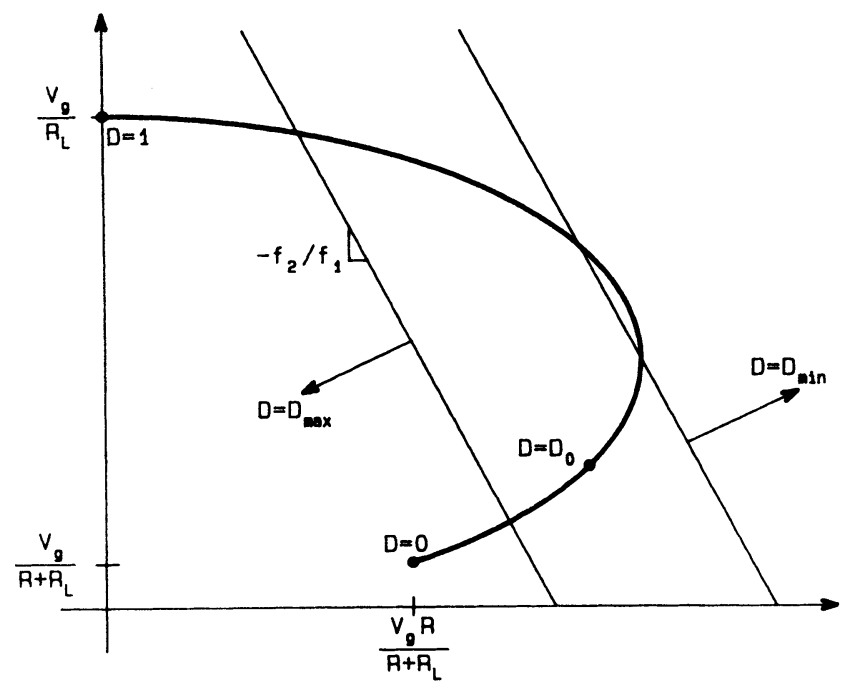

Fig. 11. The locus of saturated region equilibrium points in the state plane is a portion of an ellipse. The saturated region boundaries are superimposed. The regulator must be designed such that both saturated region equilibriun points are virtual.

Thus, the positions of the equilibrium points can be calculated without difficulty. The presence of real equilibrium points in addition to the desired quiescent operating point indicates that the regulator is not globally stable; therefore, these points must be eliminated. As an example, the equilibrium points of a two-state boost regulator were found. In addition to the quiescent operating point, one real equilibrium point existed in the unsaturated region and another in the $D=1$ saturated region. Hence, this design was not globally stable. The response could be improved by moving these equilibrium points well outside of their respective regions; they would then become virtual equilibrium points.

\subsection{Construction of Trajectories}

Knowledge of the existence and positions of the equilibrium points of the regulator yields a great deal of insight into the large-signal operation of the system; however, this knowledge is not complete. It is also necessary to calculate peak transient currents and voltages, and to verify that all possible responses are well-behaved and stable. Therefore, it is desirable to construct the system trajectories.

A number of methods exist for the analytical construction of trajectories $[8,9]$, such as the vector-field method or the method of isoclines. For second-order systems, these methods work well. However, since the dimension of the state space is equal to the number of states of the system, these analytical methods become impractical for regulators with many states, and it becomes necessary to resort to computer simulation. The state equations describing the transient response of the regulator, Eq. (10), are easily implemented

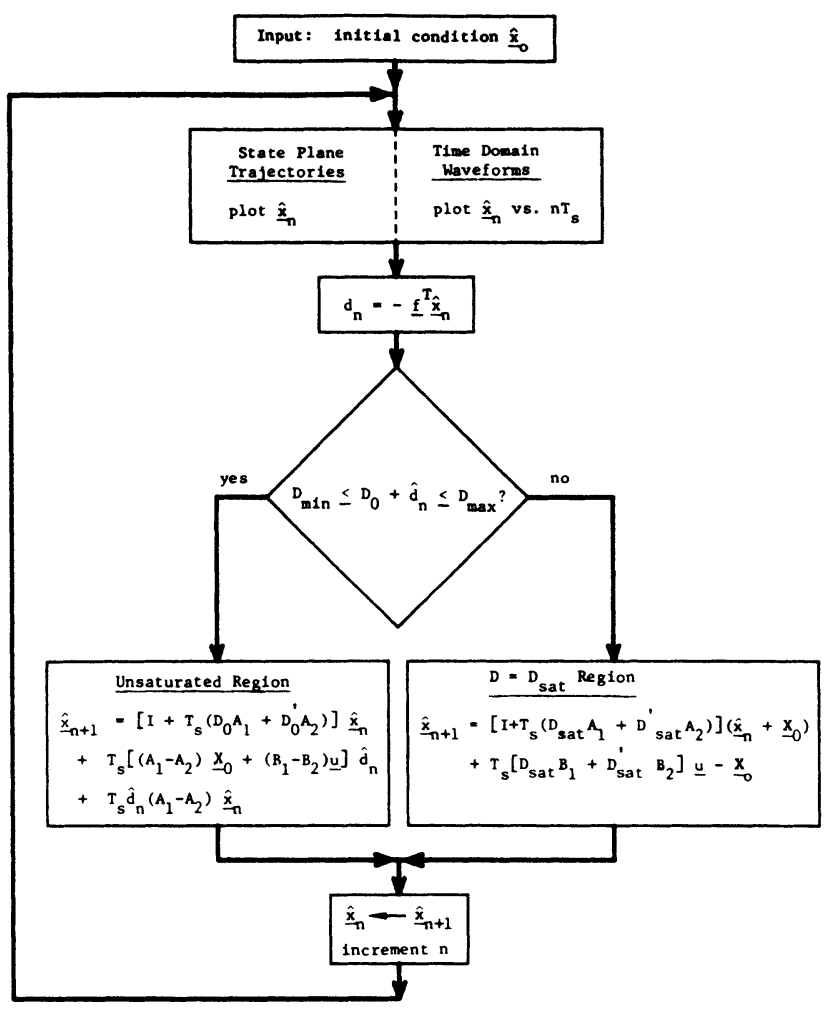

Fig. 12. Flowchart of the computer program for the calculation of trajectories and time domain transient waveforms.

on most computers. The state-plane trajectories illustrated in this paper were plotted by a small desktop computer programmed in BASIC.

Figure 12 contains the flowchart of the program. The initial state $\hat{x}_{0}$ of the system is given as input, and then the computer iteratively calculates $\hat{x}_{n}$, the values of the state variables at the switching instants $t=\mathrm{nT}_{S}$, as follows: at the $n$-th switching interval, the duty ratio $\hat{d}_{n}$ is evaluated from Eq. (11). The region of operation is determined from Eq. (15). If the system is in the unsaturated region, then Eq. (10) is used to find $\hat{x}_{n+1}$. If the system is in one of the saturated regions, then Eq. (16) is used to find $\hat{\underline{x}}_{n+1}=\left(\underline{x}_{n+1}-\underline{x}_{0}\right)$, with $D_{\text {sat }}$ taken to be either $D_{\max }$ or $D_{\min }$, depending on the region. The program then increments $n$ and repeats the procedure. State-plane trajectories may be obtained, where the values of two of the states are plotted in a plane, or time domain waveforms may be found.

The computer-drawn state plane trajectories for the two-state boost regulator example are shown in Fig. 13. The peak values of inductor current and output voltage during any given transient are apparent. The effect of the unsaturated region real equilibrium point at $i^{*}=2.0 \mathrm{~A}, \mathrm{v}^{*}=-3.7 \mathrm{~V}$ can also be seen: some unstable solutions occur which bend away in the vicinity of the equilibrium point and head towards a large negative value of $\hat{v}$. This equilibrium point is a saddle point. As explained in the 


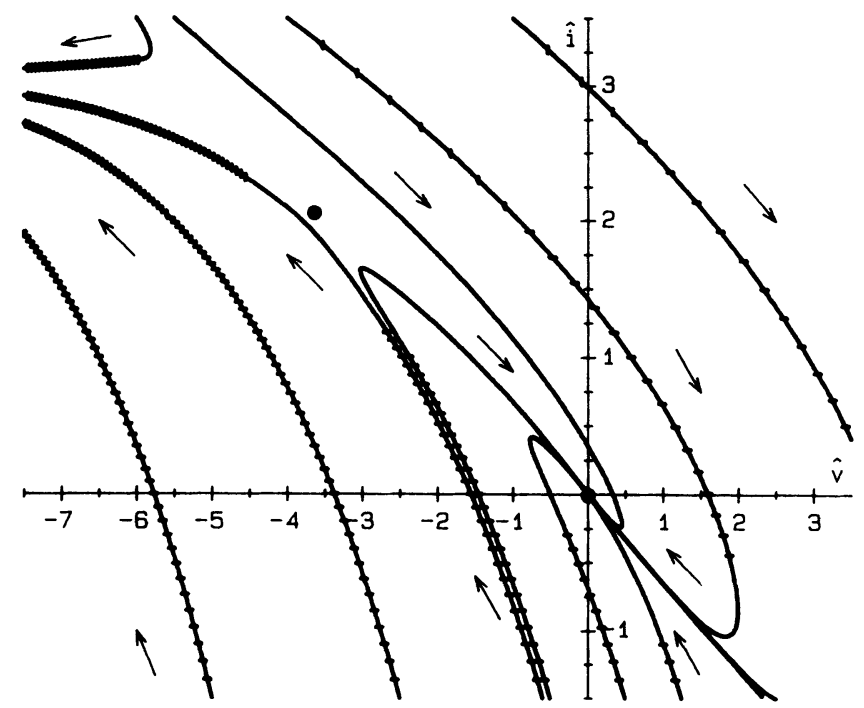

Fig. 13. Computer-predicted state-plane trajectories for the two-state boost example. Some transients converge to the desired quiescent operating point $\hat{\mathrm{x}}=0$, while others are unstable and converge to the real equilibrium in the $\mathrm{D}=\mathrm{D} \max$ saturated region. The real equilibrium point at $\mathrm{d}^{*}=.236$ is a stable point.

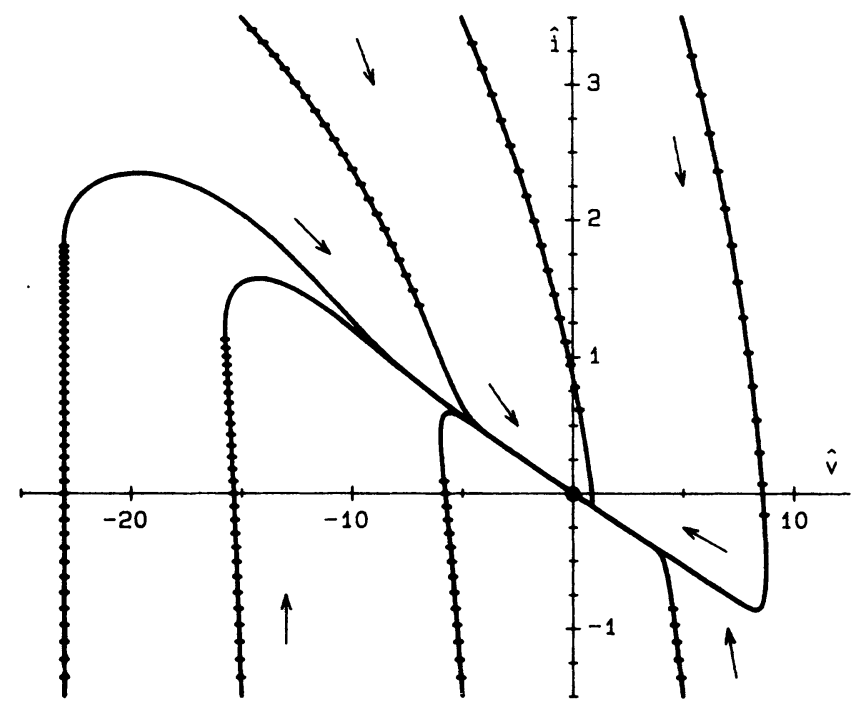

Fig. 34. The quiescent operating point of the two-state boost regulator example becomes globally stable when the ratio of $\mathrm{f}_{1}$ to $\mathrm{f}_{2}$ is increased a sufficient amount.

previous section, another real equilibrium point exists in the $D=1$ saturated region. This point is a stable equilibrium point, and all solutions which do not converge to the desired quiescent operating point converge to this additional equilibrium point.
This system may be made globally stable by sufficient increase of the ratio of current feedback to voltage feedback. The system then has four virtual equilibrium points in addition to the real quiescent operating point. The trajectories are plotted in Fig. 14 for the values $f_{1}=0.8$, $\mathrm{f}_{2}=0.08$. It can be seen that all solutions converge to the point $\hat{\mathrm{i}}=0, \hat{\mathrm{v}}=0$, and a well behaved, globally stable response is obtained.

Transient waveforms may also be plotted vs. time. This was done for the boost with integrator example in Figs. 5-7. In this way, the actual response of systems with more than two states may be predicted, and the existence or absence of unstable solutions verified.

Thus, the nonlinear models of Section 2 may be used to investigate the large-signal response of a switching regulator. Equilibrium points may be calculated analytically, and positioned properly to obtain a well-behaved response. The actual state-plane trajectories or time-domain waveforms may be calculated easily by a small desktop computer; this allows the informed design of the regulator and ensures a globally stable system.

\section{CONCLUSIONS}

Because the small-signal switching regulator models are linear and hence easily applied to most practical design problems, and because of the insight they afford into the operation of the regulator, they are indispensable tools for the design of a switching regulator. However, because of the small-signal assumption, these models do not describe the behavior of the regulator during large transients. As evinced by the example in Section 1.1 , it is possible for a regulator to be stable for small perturbations but not for all large perturbations. A design of this type is unreliable.

It is of interest, therefore, to formulate models which are valid for large signals and to determine the nature of these large-signal instabilities. It would then be possible to ensure that the large-signal transient response is wel1behaved. A set of large-signal models is described in Section 2. The key linear-ripple approximation of the small-signal state-space averaging method $[1,2,4,5]$ is employed, but no small-signal assumption is made. The effect of the saturation of the pulse-width modulator is also included, as its influence is substantial.

The equilibrium points of a system are prominent features of its state plane portrait, and the knowledge of their number and positions can yield a great deal of insight into the largesignal system behavior. The equilibrium points of switching regulators are studied in Section 3.1. Analytical expressions are found which may be used to place the equilibrium points at suitable locations in the state plane, thereby improving the large-signal transient response. This is demonstrated for the example of a two- 
stable boost regulator. It is found that this egulator has four equilibrium points in addition to the quiescent operating point. Only two of these points may have a serious detrimental effect on the response, however. With proper circuit design, this example may be rendered globally stable.

The actual state-plane trajectories or time-domain transient response may be found. This is easily accomplished by the computer evaluation of the models of Section 2. In this way, the existence of unstable solutions may be observed, and peak values of transient response waveforms calculated.

A number of effects have been neglected here. Additional modes of operation may exist, such as transient discontinuous conduction mode or current limiting modes. Also, more analysis is possible, such as the prediction of $1 \mathrm{imit}$ cycles and the analytical estimation of stability regions. Nonetheless, the most basic aspects of the nonlinear phenomena which occur have now been described, and the informed large-signal design of most switching regulators is now possible.

\section{REFERENCES}

[1] R. D. Middlebrook and Slobodan Cuk, "A General Unified Approach to Modelling Switching-Converter Power Stages," IEEE Power Electronics Specialists Conference, 1976 Record, pp. 18-34 (IEEE Publication 76CH1084-3 AES); also International J. of Electronics, vol. 42, no. 6, pp. 521-550, June 1977.

[2] Farhad Barzegar, Slobodan Cuk, and R. D. Middlebrook, "Using Small Computers to Model and Measure Magnitude and Phase of Regulator Transfer Functions and Loop Gain," Proc. Eighth International Solid-State Power Conversion Conference (Powercon 8), Dallas, Texas, April 27-30, 1981, pp. H-1.1-28.

[3] Arthur R. Brown and R. D. Middlebrook, "Sampled-Data Modeling of Switching Regulators," Power Electronics Specialists Conference, 1981 Record, Boulder, Colorado, June 1981, pp. 349-369, (IEEE Publication 81CH1652-7).

[4] Slobodan Cuk, "Modelling, Analysis, and Design of Switching Converters," PhD thesis, California Institute of Technology, November 1976.

[5] R. D. Middlebrook and Slobodan Cuk, Advances in Switched-Mode Power Conversion, Vol. I, IESLACo, 1981.

[6] K. Harada and T. Nabeshima, "Large-Signal Transient Response of a Switching Regulator," IEEE Power Electronics Specialists Conference, 1981 Record, Boulder, Colorado, June 1981, pp. 388-394 (IEEE Publication 81CH1652-7).
[7] D. B. Edwards and T. K. Caughey, "Global Analysis of a Buck Regulator," IEEE Power Electronics Specialists Conference, 1978 Record, Syracuse, New York, June 1978, pp. 12-16 (IEEE Publication 78CH1337-5 AES).

[8] M. Vidyasagar, Nonlinear Sustems Analysis, Prentice-Hal1, 1978.

[9] J. J. Stoker, Nonlinear Vibrations, John Wiley and Sons, 1950.

[10] Robert W. Erickson and R. D. Middlebrook, "Origins of Harmonic Distortion in Switching Amplifiers," Proc. International PCI/MOTORCON ' 82 Conference, San Francisco, California, March 29-31, 1982.

[11] Robert W. Erickson and R. D. Middlebrook, "Large-Signal Modelling and Analysis of Distortion in Switching Amplifiers," Power Electronics Group, California Institute of Technology, January 1982. 